\title{
A novel biomarker, active whole skeletal total lesion glycolysis (WS-TLG), as a quantitative method to measure bone metastatic activity in breast cancer patients
}

\author{
Mitsuru Koizumi ${ }^{1}$ (D) $\cdot$ Kazuki Motegi $^{1} \cdot$ Takuro Umeda $^{1}$
}

Received: 27 January 2019 / Accepted: 5 April 2019 / Published online: 13 April 2019

(c) The Author(s) 2019

\begin{abstract}
Objective There is no good response evaluation method for skeletal metastasis. We aimed to develop a novel quantitative method to evaluate the response of skeletal metastasis, especially lytic lesions, for treatment.

Methods A method to measure active bone metastatic burden quantitatively using F-18 fluorodeoxyglucose positron emission tomography with computed tomography (FDG-PET/CT) in breast cancer patients, whole skeletal total lesion glycolysis (WS-TLG), a summation of each skeletal lesion's TLG, was developed. To identify active bone lesions, a tentative cutoff value was decided using FDG-PET/CT in 85 breast cancer patients without skeletal metastasis and 35 with skeletal metastasis by changing the cutoff value. Then, the WS-TLG method was evaluated by comparing to PET Response Criteria in Solid Tumor (PERCIST) or European Organization for Research and Treatment of Cancer (EORTC) criteria for only bone in 15 breast cancer patients with skeletal metastasis who were treated.

Results A cutoff value of the standardized uptake value (SUV) $=4.0$ gave $91 \%$ (77/85) specificity and 97\% (34/35) sensitivity. We decided on SUV $=4.0$ as a tentative cutoff value. Skeletal metastases of lytic and mixed types showed higher WS-TLG values than those of blastic or intertrabecular types, although statistical significance was not tested. All 15 patients showed agreement with PERCIST or EORTC in the therapeutic bone response.

Conclusion This quantitative WS-TLG method appears to be a good biomarker to evaluate skeletal metastasis in breast cancer patients, especially lytic or mixed types. Further clinical studies are warranted to assess the clinical values of this new WS-TLG method.
\end{abstract}

Keywords Breast cancer $\cdot$ Skeletal metastasis $\cdot$ FDG-PET/CT $\cdot$ Bone tumor burden $\cdot$ TLG

\section{Introduction}

Breast cancer often metastasizes to the skeleton [1]. Many diagnostic techniques have been employed to detect skeletal metastases and to measure their therapeutic effect [2]. Historically, $\mathrm{x}$-ray has been used, but it has extremely low detectability. X-ray computed tomography (CT) has higher detectability than $\mathrm{X}$-ray but lower detectability than modern techniques such as magnetic resonance imaging (MRI) and positron emission tomography (PET) using F-18 fluorodeoxyglucose (FDG) or sodium F-18 fluoride (NaF). MRI and

Mitsuru Koizumi

mitsuru@jfcr.or.jp

1 Department of Nuclear Medicine, Cancer Institute Hospital, 3-8-31 Ariake, Koto-ku, Tokyo 135-8555, Japan
PET are now the best techniques to detect skeletal metastases [3]. Another approach to measure bone metastatic burden is to use serum or urine bone metabolic markers [4]. Bone scintigraphy with the aid of artificial intelligence (AI) (Exnibone or Bone-Navi) is a method to quantitate osteoblastic activity using planar images. This method is clinically successful in osteoblastic metastases such as prostate cancer [5-8]. However, an accurate quantitative method to measure bone metastatic burden in breast cancer patients has not been established.

A method to measure bone metastatic activity using sodium fluoride $(\mathrm{NaF}) \mathrm{PET} / \mathrm{CT}$ in prostate cancer has been reported [9]. We adopted this method to bone scintigraphy with single photon emission tomography with CT (SPECT/ CT) in prostate cancer [10]. 
These tomographic methods are useful to measure osteoblastic osseous metastasis, however, are not useful to measure osteolytic osseous metastasis.

FDG uptake by osteolytic lesions is a good marker to measure [2, 11, 12], and FDG-PET/CT is now one of the main methodologies to detect and to measure skeletal metastasis.

Therefore, we tried to develop a method to measure active bone metastatic burden quantitatively using FDG-PET/CT in breast cancer patients. Briefly, (1) bone element is identified by CT attenuation. (2) Increased FDG uptake areas in the bone element are identified using a cutoff level, and these areas are regarded as the bone lesions. (3) Then, the product of lesion volume and mean FDG uptake in the lesion is calculated. This is the active bone metastatic index of a lesion: total lesion glycolysis (TLG) of one lesion. (4) The sum of TLGs of all skeletal lesions is performed; this is the active whole skeletal total lesion glycolysis (WS-TLG) of the study. This active WS-TLG method quantifies osseous tumor burden (FDG avidity and bone tumor volume).

\section{Patients and methods}

\section{Patients}

This is a retrospective observational study. Local ethical approval of this study was obtained (2018-1051), and informed consent was waived. All patients were diagnosed and treated for breast cancer at Cancer Institute Hospital, Ariake, Koto-ku, Tokyo, Japan.

After the local ethical approval was obtained, patient recruitment was started. Three groups of patients were selected retrospectively using our FDG-PET/CT study log.

First: eighty-five breast cancer patients without skeletal metastasis were collected from February 2018 to April 2018. The threshold or cutoff level was determined based on their data.

Second: thirty-five breast cancer patients with newly diagnosed skeletal metastasis were collected from November 2014 to April 2018. The sensitivity was calculated using FDG-PET/CT data of these patients.

Third: fifteen breast cancer patients with newly developed skeletal metastasis and at least 2 or more follow-up FDG-PET/CT studies from July 2013 to October 2018 were included.

The diagnosis of skeletal metastasis was established as follows: when the findings of FDG and CT were typical for skeletal metastasis (multiple intraosseous lesions, FDG avid, and obvious CT morphologic changes), the clinical diagnosis was established. When the patients had an intertrabecular type bone lesion or solitary osseous lesion and MRI showed the positive findings, the clinical diagnosis was established.
For the patients whom the clinical diagnosis was not established even by MRI, bone biopsies were performed. The positive bone biopsy result meant the pathological diagnosis of skeletal metastasis. The patients with negative results of bone biopsy or lesions not suitable for bone biopsy underwent further follow-up studies, and the results showed the diagnosis of typical skeletal metastasis. The patients who did not have skeletal metastasis were diagnosed by normal bone FDG and CT findings and clinical follow-up for more than 6 months.

Thirty-five patients were diagnosed with skeletal metastasis. The diagnosis was established by FDG and CT in 27 of the 35 patients, by MRI in 2 patients, and by bone biopsy in 4 patients, and 2 patients were diagnosed by follow-up studies after biopsy failure.

\section{FDG-PET/CT study}

We used routinely performed FDG-PET/CT studies for this investigation. Patients fasted for at least $6 \mathrm{~h}$ before being injected with $4 \mathrm{MBq} / \mathrm{kg}$ FDG and then whole-body image acquisition started at $60 \mathrm{~min}$ later from the top of the skull to the mid-thigh using a Discovery 610 PET/CT scanner (GE, USA) or Discovery IQ PET/CT scanner (GE, USA). Emission data were acquired for 1-3 min per bed position. Discovery 610 PET images were reconstructed using threedimensional ordered-subsets expectation-maximization with point-spread function (OSEM + PSF); 3 iterations, 16 subsets with a 4-mm Gaussian filter, a $192 \times 192$ matrix $(2.6 \mathrm{~mm} /$ pixel). Discovery IQ PET images were reconstructed using three-dimensional ordered-subsets expectation-maximization with point-spread function (OSEM + PSF); 4 iterations, 12 subsets with a 4-mm Gaussian filter, a $192 \times 192$ matrix (2.6 mm/pixel).

Whole-body CT scanning proceeded under the following parameters: $120 \mathrm{kV}$; auto exposure control system (noise level: SD 10); $512 \times 512$ matrix; beam pitch, $3.75 \mathrm{~mm} \times 16$-row mode.

\section{Active whole skeletal total lesion glycolysis (WS-TLG)}

We considered that following equation gave active bone tumor burden or active WS-TLG: first, bone component was extracted by CT. A CT attenuation above 152 Hounsfield Units (HU) was regarded as the bone component [13]. Metal artifact areas and calcified areas were also included; therefore, these areas were deleted manually. Then the following formula was used to calculate active WS-TLG:

Active WS-TLG $=\sum$ (volume above threshold, $\mathrm{mL}$ ) $x$ (mean standardized uptake value of the lesion). 
Figure 1 shows the process to obtain active WS-TLG. These processes were performed semi-automatically by a software GI-BONE (AZE, Kanagawa, Japan).

\section{Deciding the threshold value}

The maximum of Standardized Uptake Value (SUVmax) was measured in middle thoracic vertebra (Th7), 3rd lumbar vertebra (L3), sternum, rib, and ilium in 41 FDG-PET/CT studies in the breast cancer patients without skeletal metastasis. These regions were selected based on the frequency of skeletal metastasis [14]. A tentative threshold value was determined by the measurement of normal bone SUVmax distribution. Then, WS-TLG was calculated for 85 patients without skeletal metastasis and 35 patients with skeletal metastasis changing cutoff levels.

The negative rates, that is, any number above 0 , in 85 patients without skeletal metastasis (specificity) and the positive rates in 35 patients with skeletal metastasis (sensitivity) were calculated. We decided a cutoff value by comparing specificity and sensitivity.

\section{Sensitivity and specificity}

Sensitivity and specificity of WS-TLG were calculated using the cutoff level in 35 patients with newly developed skeletal metastasis and 85 patients without skeletal metastasis. In patients with skeletal metastasis, WS-TLG value differences were investigated based on CT types of skeletal metastasis.

\section{WS-TLG in patients with FDG follow-up}

WS-TLG changes in each patient were compared with the results using PET Response Criteria in Solid Tumor (PERCIST) or European Organization for Research and Treatment of Cancer (EORTC) criteria [15-17].

We analyzed skeletal metastasis by PERCIST or EORTC regardless of other metastatic lesions. In PERCIST, up to two osseous lesions with highest FDG uptake (the peak standardized uptake value corrected for lean body mass: SULpeak) were chosen and followed on subsequent studies. When the therapeutic regimens changed, new sets of osseous lesions were selected.

When initial FDG uptake was low and the PERCIST could not be applied, EORTC criteria were applied to up to two osseous lesions regardless of other metastases. For EORTC, the sum of SUVmax was used, and for PERCIST, SULpeak values were used for evaluation [17]. Both analyses were performed by PET VCAR for Advantage Workstation (GE, USA).

The PERCIST metabolic response categories were complete metabolic response (CMR); no FDG-avid tumor visible (SULpeak was decreased to the background level), partial metabolic response (PMR); target lesions must
Fig. 1 The process to obtain whole skeletal total lesion glycolysis (WS-TLG) was shown. Whole-body FDG-PET image (anterior view of maximum intensity projection; MIP) was taken (a). Bone areas were decided using CT attenuation (more than $152 \mathrm{HU}$, red areas) (b). The lesions above cutoff level (SUV > 4.0) were chosen (red lesions) (c). Each lesion's TLG was calculated by the product of the volume and mean SUV of each bone lesion, and then, the summation of TLG values of whole skeletal lesions gave the WS-TLG value

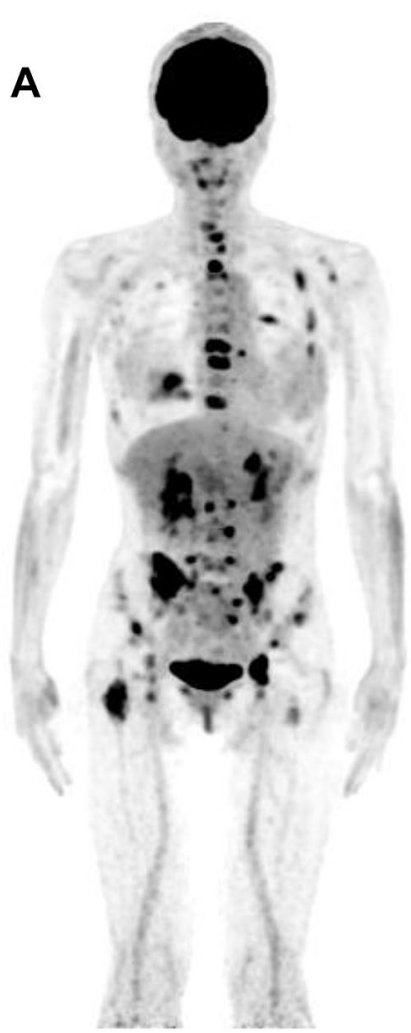

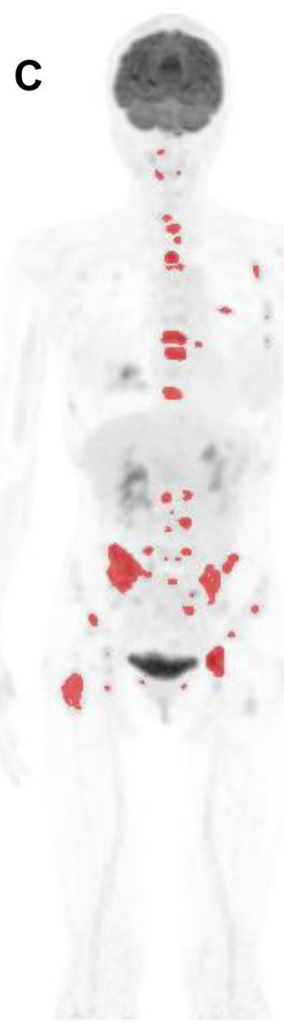


show SULpeak decrease of greater than or equal to $30 \%$ and of at least 0.8 SUL units, progressive metabolic disease (PMD); SULpeak of target lesions must show an increase of greater than or equal to $30 \%$ and an increase of at least $0.8 \mathrm{SUL}$ units or development of one or more new lesions; and stable metabolic disease (SMD); a response between PMR and PMD [18].

The EORTC response was divided into four categories: CMR was complete resolution of FDG uptake within all lesions, PMR was a reduction in the sum of SUVmax of at least $25 \%$ decrease after more than 1 treatment cycle, PMD was an increase of at least $25 \%$ in the sum of SUVmax or a new FDG-avid lesion, and SMD was response between PMR and PMD [17].

\section{Results}

\section{Search for cutoff level}

Table 1 shows the SUVmax values in various skeletal sites in 41 patients without skeletal metastasis. The SUVmax values were variables depending on sites. Among them, vertebral SUVmax values were higher than other sites. In vertebral sites, SUV max + 3SD was around 3.5. We regarded the $\mathrm{SUV}=3.5$ as the starting point to search for the optimal cutoff level.

Then, WS-TLG calculations were performed on 85 patients without and 35 patients with skeletal metastasis by changing cutoff levels $(3.5,4.0$, and 4.5$)$.

Table 2 shows WS-TLG specificities in patients without skeletal metastasis and sensitivities in skeletal metastasis with various cutoff levels $(3.5,4.0$, and 4.5). Since SUV $=4.0$ gave the specificity of $91 \%$ and the high sensitivity (97\%) in patients with skeletal metastasis, we decided on SUV $=4.0$ as the tentative cutoff level.
Table 2 WS-TLG values in breast cancer patients without or with bone metastasis with the function of cutoff value

\begin{tabular}{|c|c|c|c|c|}
\hline & & \multicolumn{3}{|c|}{ SUV $^{\mathrm{c}}$ Cutoff value } \\
\hline & & 3.5 & 4.0 & 4.5 \\
\hline $\begin{array}{l}\text { WS-TLG }{ }^{\mathrm{a}} \text { in } \\
\text { patients without } \\
\text { bone metastasis }\end{array}$ & Negative & $69^{b} / 85$ & $77 / 85$ & $80 / 85$ \\
\hline (85 patients) & Specificity (\%) & $81 \%$ & $91 \%$ & $94 \%$ \\
\hline $\begin{array}{l}\text { WS-TLG in } \\
\text { patients with } \\
\text { bone metastasis }\end{array}$ & Positive & $34 / 35$ & $34 / 35$ & $32 / 35$ \\
\hline (35 patients) & Sensitivity (\%) & $97 \%$ & $97 \%$ & $91 \%$ \\
\hline
\end{tabular}

${ }^{\mathrm{a}}$ WS-TLG (whole skeletal total lesion glycolysis)

${ }^{\mathrm{b}}$ Number of patients

${ }^{\mathrm{c}} \mathrm{SUV}$ (standardized uptake value)

\section{Sensitivity and specificity}

The specificity was $91 \%(77 / 85)$ in the 85 breast cancer patients without skeletal metastasis.

The sensitivity was $97 \%$ (34/35) in 35 breast cancer patients with newly developed skeletal metastasis.

The WS-TLG values (mean \pm SD) according to type of skeletal metastases (Table 3), were $30.9 \pm 45.6$ for the osteoblastic type, $30.9 \pm 43.3$ for the intertrabecular type (invisible type), $208.7 \pm 326.3$ for the osteolytic type, and $393.7 \pm 680.0$ for the mixed type, respectively. Statistical analysis was not performed because the number of patients was small. However, WS-TLG tended to be low in osteoblastic and intertrabecular types, and high in osteolytic and mixed types.

\section{Follow-up}

WS-TLG in patients with skeletal metastasis who were followed up by repeated FDG study.

A total of 15 breast cancer patients with skeletal metastasis and pre-treatment FDG-PET/CT studies, and at least two follow-up FDG-PET/CT studies were selected randomly.
Table 1 FDG SUVmax values in various bones in breast cancer patients without skeletal metastasis

\begin{tabular}{lllllll}
\hline Bone site & \multicolumn{3}{l}{ SUVmax value } & & Mean +2SD & Mean + 3SD \\
\cline { 2 - 4 } & Mean & SD & Minimum & Maximum & & \\
\hline Sternum & 1.43 & 0.33 & 0.86 & 2.17 & 2.09 & 2.42 \\
Rib & 1.27 & 0.43 & 0.78 & 2.37 & 2.13 & 2.56 \\
Th7 & 2.29 & 0.36 & 1.66 & 2.97 & 3.01 & 3.37 \\
L3 & 2.33 & 0.36 & 1.56 & 3.26 & 3.05 & 3.41 \\
Ilium & 1.66 & 0.31 & 1.09 & 2.33 & 2.28 & 2.59 \\
\hline
\end{tabular}

${ }^{\mathrm{a}}$ Values were measured on FDG-PET/CT images in breast cancer patients without skeletal metastasis SUVmax: maximum of standardized uptake value 
Table 3 WS-TLG values applying 4.0 cutoff value in breast cancer patients with newly diagnosed skeletal metastasis

\begin{tabular}{lllllr}
\hline CT type of bone metastasis & No. patients & $\begin{array}{l}\text { WS-TLG }^{\mathrm{a}}- \\
\text { positive }\end{array}$ & $(\%)$ & Mean WS-TLG & SD \\
\hline Blastic type & 5 & 5 & $100 \%$ & 30.9 & 45.6 \\
Intertrabecular type & 6 & 5 & $83 \%$ & 30.9 & 43.3 \\
Lytic type & 12 & 12 & $100 \%$ & 208.7 & 326.3 \\
Mixed type & 12 & 12 & $100 \%$ & 393.7 & 680.0 \\
Total & 35 & 34 & $97 \%$ & 216.2 & 454.7 \\
\hline
\end{tabular}

${ }^{\mathrm{a}}$ WS-TLG; whole skeletal total lesion glycolysis
As shown in Table 4 (1, 2, and 3), 4 patients had favorable bone responses (WS-TLG) to therapy, 5 patients had progressive bone responses, and 6 patients had fluctuating responses.

In the favorable WS-TLG response group (Table 4), all 4 patients showed decreasing or very low at pre-therapy value (0) and no increase at follow-up studies. WS-TLG values and bone PERCIST or bone EORTC response were concordant.

In the progressive WS-TLG response group (Table 5), WS-TLG changes and bone PERCIST or EORTC agreed in all 5 patients. A representative case (Case 9) is shown in Fig. 2. She underwent FDG-PET/CT due to an elevation of a tumor marker (CA15-3) after her surgery for breast cancer. Oligo bone metastases were noted in bilateral iliac bones (A), and her treatment was changed to hormone therapy. The first follow-up FDG-PET/CT study showed an increased number of skeletal metastases (B), and then her skeletal metastases progressed at the second follow-up (C).
In fluctuating WS-TLG response group (Table 6), WSTLG changes and bone PERCIST were concordant in all 6 patients. A representative case (Case 11) is shown in Fig. 3. She noticed shortness of breath 10 years after her right breast surgery. FDG-PET/CT was performed, and pleural dissemination and multiple osteolytic skeletal metastases were diagnosed (A). She was prescribed hormone therapy after the initial FDG-PET/CT study. The first follow-up FDG-PET/CT was performed after 6 months of hormone therapy and showed improvement in pleural dissemination and skeletal metastases (B). Since her tumor marker (CA15-3) was elevated (42.3 U/mL), a second follow-up FDG-PET/CT was performed. FDG uptake in skeletal metastasis had increased and was judged to be progressive disease (C).
Table 4 Comparison of WS-TLG and PERCIST or EORTC in breast cancer skeletal metastatic lesions; improved group

\begin{tabular}{|c|c|c|c|c|c|c|c|}
\hline Case No. & FDG study ${ }^{a}$ & Age & WS-TLG & PERCIST & EORTC & CT type & Comment \\
\hline \multirow[t]{3}{*}{1} & Pre Tx & 49 & 11.6 & & & Mixed & \\
\hline & $3 \mathrm{M}$ & & 0 & CMR & & Blastic & \\
\hline & $1 \mathrm{Y} 7 \mathrm{M}$ & & 0 & CMR & & Normal & \\
\hline \multirow[t]{4}{*}{2} & Pre $T x$ & 70 & 0 & NA & & Intertrabecular & \\
\hline & $1 \mathrm{Y} 1 \mathrm{M}$ & & 0 & & SMD & Blastic & \\
\hline & $2 \mathrm{Y} 8 \mathrm{M}$ & & 0 & & SMD & Blastic & \\
\hline & $3 \mathrm{Y} 5 \mathrm{M}$ & & 0 & & SMD & Blastic & \\
\hline \multirow[t]{4}{*}{3} & Pre Tx & 57 & 22.0 & & & Mixed (lytic predominant) & \\
\hline & $4 \mathrm{M}$ & & 0.5 & PMR & & Blastic & \\
\hline & $8 \mathrm{M}$ & & 0 & CMR & & Blastic & \\
\hline & $1 \mathrm{Y}$ & & 0 & CMR & & Blastic & \\
\hline \multirow[t]{4}{*}{4} & Pre $T x$ & 44 & 22.4 & & & Blastic, faint & \\
\hline & $7 \mathrm{M}$ & & 0 & CMR & & Blastic, intense & \\
\hline & $1 \mathrm{Y} 7 \mathrm{M}$ & & 0 & CMR & & Blastic, intense & \\
\hline & $2 \mathrm{Y}$ & & 0 & CMR & & Blastic, intense & \\
\hline
\end{tabular}

PERCIST PET response criteria in solid tumor, EORTC criteria developed by the European Organization for Research and Treatment of Cancer, $T x$ treatment, $N A$ not applicable, $C M R$ complete metabolic remission, $P M R$ partial metabolic response, $S M D$ stable metabolic disease, $P M D$ progressive metabolic disease, $W S-T L G$ whole skeletal total lesion glycolysis

${ }^{\text {a }}$ Time after commencement of therapy for skeletal metastasis 
Table 5 Comparison of WS-TLG and PERCIST or EORTC in breast cancer skeletal metastatic lesions; progressed group

\begin{tabular}{|c|c|c|c|c|c|c|c|}
\hline Case No. & FDG study ${ }^{a}$ & Age & WS-TLG & PERCIST & EORTC & CT type & Comment \\
\hline \multirow[t]{3}{*}{5} & Pre Tx & 58 & 2.8 & & & Blastic & \\
\hline & $1 \mathrm{Y}$ & & 76.7 & PMD & & Mixed & \\
\hline & $2 \mathrm{Y}$ & & 532.5 & PMD & & Mixed & \\
\hline \multirow[t]{3}{*}{6} & Pre Tx & 57 & 0.4 & & & Intertrabecular & \\
\hline & $9 \mathrm{M}$ & & 15.3 & PMD & & Mixed & \\
\hline & $1 \mathrm{Y} 6 \mathrm{M}$ & & 509.8 & PMD & & Mixed & \\
\hline \multirow[t]{3}{*}{7} & Pre $T x$ & 65 & 0 & NA & & Intertrabecular & \\
\hline & $5 \mathrm{M}$ & & 6.6 & & PMD & Intertrabecular & \\
\hline & $11 \mathrm{M}$ & & 23.2 & & PMD & Intertrabecular & \\
\hline \multirow[t]{3}{*}{8} & Pre Tx & 55 & 0 & NA & & Intertrabecular & \\
\hline & $10 \mathrm{M}$ & & 1.0 & & PMD & Blastic & \\
\hline & $1 \mathrm{Y} 7 \mathrm{M}$ & & 234.8 & & PMD & Blastic & \\
\hline \multirow[t]{3}{*}{9} & Pre Tx & 71 & 0.2 & & & Intertrabecular to lytic & \\
\hline & $1 \mathrm{Y} 5 \mathrm{M}$ & & 199.2 & PMD & & Blastic & \\
\hline & $1 \mathrm{Y} 9 \mathrm{M}$ & & 889.4 & PMD & & Blastic & \\
\hline
\end{tabular}

PERCIST PET response criteria in solid tumor, EORTC criteria developed by the European Organization for Research and Treatment of Cancer, $T x$ treatment, $N A$ not applicable, CMR complete metabolic remission, $P M R$ partial metabolic response, $S M D$ stable metabolic disease, $P M D$ progressive metabolic disease, $W S-T L G$ whole skeletal total lesion glycolysis

${ }^{a}$ Time after commencement of therapy for skeletal metastasis
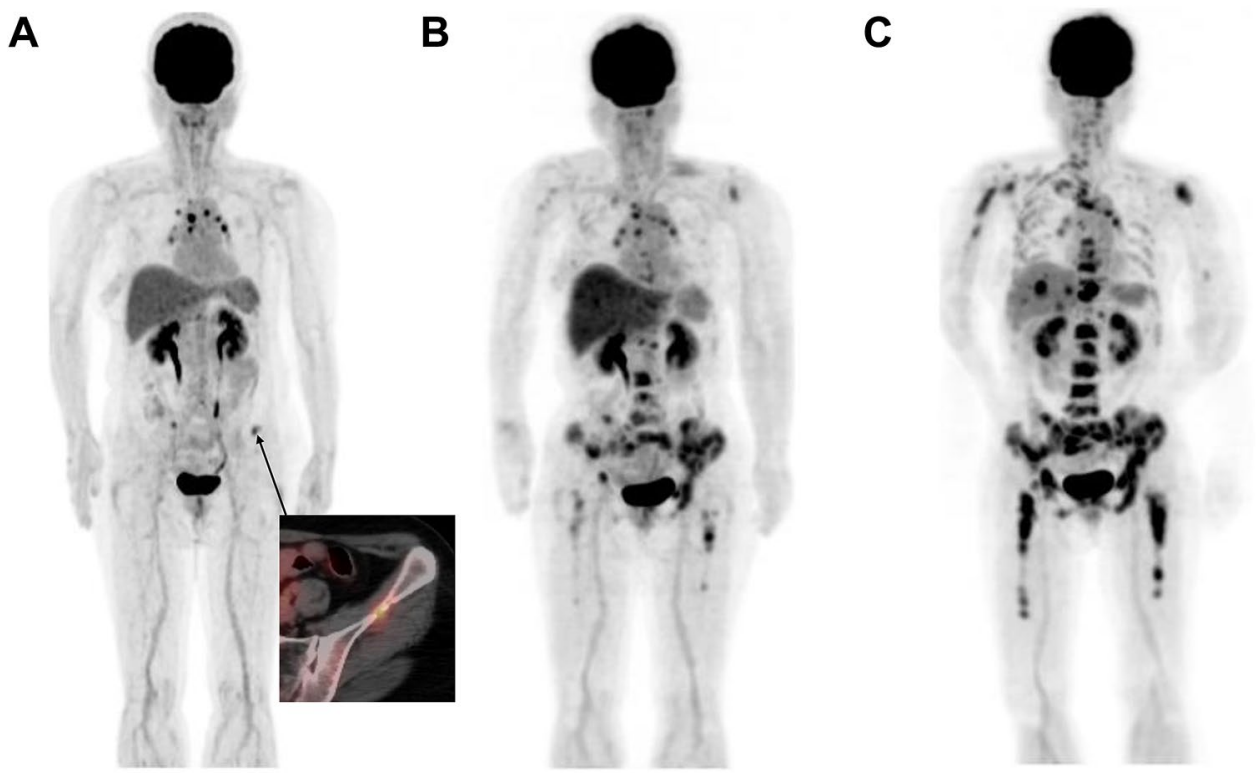

Fig. 2 This is Case No. 9 in Table 5. Anterior views of FDG-PET MIP images at the time of diagnosis (a) and first (b), and second (c) follow-up studies are shown. She received FDG-PET/CT due to an elevation of a tumor marker (CA153) after her surgery of breast cancer. Oligo bone metastasis was noticed in bilateral iliac bones (a), and hormone therapy had begun. First follow-up FDG-PET/CT study showed an increased number of skeletal metastasis (b), then her skeletal metastasis further progressed at second follow-up (c). Her initial WS-TLG value was 0.2 , and the value increased rapidly to 199.2 at first follow-up, and 889.4 at second follow-up

\section{Discussion}

The bone is the most common site to which breast cancer metastasizes. Assessment of the change in tumor burden is an important feature of clinical evaluation of cancer therapeutics. However, oncologists often find difficulty in evaluating the therapeutic effect on skeletal metastasis. Historically, assessment methods of tumor response criteria developed by International Union Against Cancer
(UICC) and World Health Organization (WHO) have been used [2]. They were based on plain x-ray and bone scintigraphy. Recent criteria developed by the Response Criteria in Solid Tumors (RECIST 1.1) stated that (1) Bone scan, PET scan, or plain films are not considered adequate imaging techniques to measure bone lesions. (2) Lytic bone lesions or mixed lytic-blastic lesions, with identifiable soft tissue components, that can be evaluated by cross sectional imaging techniques such as CT or MRI can be considered as measurable lesions if the soft tissue component meets 
Table 6 Comparison of WS-TLG and PERCIST or EORTC in breast cancer skeletal metastatic lesions; fluctuated group

\begin{tabular}{|c|c|c|c|c|c|c|c|}
\hline Case No. & FDG study ${ }^{\mathrm{a}}$ & age & WS-TLG & PERCIST bone & EORTC bone & CT type & Comment \\
\hline \multirow[t]{3}{*}{10} & Pre Tx & 61 & 254.7 & & & Intertrabecular & \\
\hline & $4 \mathrm{M}$ & & 43.5 & PMR & & Blastic & \\
\hline & $9 \mathrm{M}$ & & 107.1 & PMD & & Blastic & \\
\hline \multirow[t]{3}{*}{11} & Pre Tx & 38 & 15.5 & & & Lytic & \\
\hline & $6 \mathrm{M}$ & & 0 & PMR & & Mixed & \\
\hline & $1 \mathrm{Y} 1 \mathrm{M}$ & & 4.6 & PMD new lesion & & Lytic, increased & \\
\hline \multirow[t]{5}{*}{12} & Pre Tx & 67 & 13.6 & & & Mixed & \\
\hline & $6 \mathrm{M}$ & & 0 & CMR & & Blastic & \\
\hline & $1 \mathrm{Y}$ & & 0 & CMR & & Blastic & \\
\hline & 1Y 6M (pre-Tx) & & 0 & CMR & & Blastic & Tx regimen change \\
\hline & $(4 \mathrm{M})$ & & 1.0 & PMD new lesion & & Mixed: blastic and intertrabecular & \\
\hline \multirow[t]{3}{*}{13} & Pre Tx & 59 & 73.4 & & & Mixed & \\
\hline & $10 \mathrm{M}($ pre-Tx) & & 80.2 & SMD & & Mixed & Tx regimen change \\
\hline & $(1 \mathrm{Y})$ & & 0.4 & CMR & & Blastic & \\
\hline \multirow[t]{5}{*}{14} & Pre Tx & 60 & 24.5 & & & Intertrabecular & \\
\hline & $8 \mathrm{M}$ & & 0 & CMR & & Mixed; intertrabecular and lytic & \\
\hline & $11 \mathrm{M}$ & & 0 & PMR & & Blastic & \\
\hline & $1 \mathrm{Y} 5 \mathrm{M}$ (pre Tx) & & 3.8 & PMD & & Blastic & Tx regimen change \\
\hline & $(1 \mathrm{Y})$ & & 12.8 & PMD & & Blastic & \\
\hline \multirow[t]{5}{*}{15} & Pre Tx & 65 & 13.3 & & & Lytic & \\
\hline & $1 Y($ pre $T x)$ & & 72.4 & PMD & & Mixed & Tx regimen change \\
\hline & $(4 \mathrm{M})$ & & 0.1 & PMR & & Mixed & \\
\hline & $(9 \mathrm{M})$ & & 10.2 & PMD & & Mixed & \\
\hline & $(1 \mathrm{Y})$ & & 14.1 & SMD & & Mixed & \\
\hline
\end{tabular}

PERCIST PET response criteria in solid tumor, EORTC criteria developed by European Organization for Research and Treatment of Cancer, $T x$ treatment, $C M R$ complete metabolic remission, $P M R$ partial metabolic response, $S M D$ stable metabolic disease, $P M D$ progressive metabolic disease, $W S-T L G$ whole skeletal total lesion glycolysis

${ }^{a}$ Time after commencement of treatment for skeletal metastasis. () means time after the change of therapy

the definition of measurability. (3) Blastic bone lesions are non-measurable [18]. This means that most skeletal metastases cannot be evaluated for therapeutic response. A group from MD Anderson Hospital proposed revised criteria for bone response [2, 19]. Other criteria are PERCIST and EORTC, in which tumor response is measured by FDG-PET uptake. Both criteria measure the change in FDG uptake in many involved organ lesions, not restricted to bone lesions. The existing criteria all use the classification of complete (metabolic) response, partial (metabolic) response, stable (metabolic), and progressive (metabolic) disease.

Apart from the response classification, we intended to make a continuous biomarker of bone tumor burden using FDG-PET/CT. We here propose a biomarker; a summation of TLGs of active bone lesions above the cutoff level. This WS-TLG method shows the glucose uptake activity in the whole skeletal lesion above threshold as a continuous number (a summation of the product of lesion volume and SUVmean of each bone lesion). We imagined this
WS-TLG value may be used, for example, serum alkaline phosphatase value or carcinoembryonic antigen level.

The cutoff level was decided using 85 patients without skeletal metastasis. We tentatively decided on SUV $=4.0$ as the cutoff value, which gave $91 \%$ of specificity (77/85) and $97 \%$ of sensitivity (34/35). Of course, cutoff value will change the study population, and this value is only a tentative one.

The WS-TLG values in patients with newly developed skeletal metastasis varied among CT types. Osteoblastic and intertrabecular types showed low values, and lytic and mixed types showed high values; no statistical evaluation could be performed because the number of patients was small. The low WS-TLG of blastic type may be the reflection of low FDG avidity of blastic metastasis [20]. The low WS-TLG of the intertrabecular type may reflect that intertrabecular type is the beginning of skeletal metastasis. High values in lytic and mixed types may indicate that this method is suitable for these CT types of skeletal metastasis. 


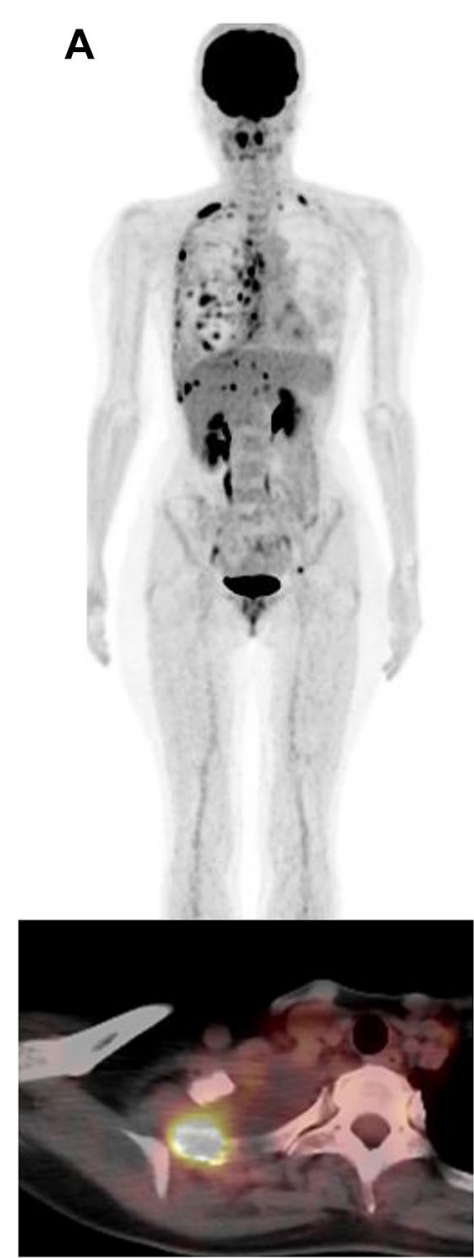

Fig. 3 This is Case No. 11 in Table 6. Anterior views of FDG-PET MIP images, and FDG-PET and CT fusion axial images at the right first rib are shown. She noticed shortness of breath 10 years after her right breast surgery. FDG-PET/CT was performed, and pleural dissemination and multiple osteolytic skeletal metastases were diagnosed (a). She was prescribed hormone therapy after the FDG-PET/ CT study (a). First follow-up FDG-PET/CT was performed after

In this study, we compared WS-TLG with PERCIST or EORTC for bone metastasis. Very good agreement was achieved in all 15 patients.

The limitations of this study are that this is the first study of WS-TLG regarding osseous metastases from cancer. WS-TLG only gives the quantitative FDG activity restricted to bone area regardless of other organs. That is, when a patient who is receiving chemotherapy for metastatic bone lesions shows improvement in bone lesions but other new (for example, new pulmonary metastasis) lesions develop, the patient's WS-TLG will improve but the total judgement of the chemotherapeutic effect is progression of disease due to the development of a new pulmonary lesion. This point can be interpreted as both an advantage (this method can evaluate bone lesions
6 months of hormone therapy and showed improvement in pleural dissemination and skeletal metastases (b). Because her tumor marker (CA15-3) was elevated (42.3 U/ml), second follow-up FDG-PET/CT was performed. FDG uptake in skeletal metastasis has increased and was judged to be progressive disease (c). Her initial WS-TLG value was 15.5 , and the value was 0 at first follow-up and 4.6 at second follow-up FDG-PET/CT study

separately) and disadvantage (this method cannot evaluate other organs).

Three patients (one in the improved group and two in the progressed group) in follow-up showed WS-TLG $=0$ at the initial assessment. The percentage of FDG non-avid skeletal metastasis differed between newly diagnosed 35 patients $(1 / 35)$ and follow-up 15 patients (3/15). We thought that FDG non-avid skeletal metastasis might be inadequate to evaluate by WS-TLG; therefore, we intentionally included FDG non-avid patients in the follow-up group. Although 3 patients whose skeletal lesions were FDG non-avid (WS$\mathrm{TLG}=0$ ) were successfully evaluated, there is still the possibility that FDG study including WS-TLG is not suitable to evaluate the therapeutic effect or clinical response in FDG non-avid skeletal lesions. 
Another limitation is different performance of each PET/ CT machine. Since SUV values obtained by old PET/CT machines differ from those by new machines and WS-TLG is calculated based on SUV value, we cannot directly compare the WS-TLG values between old and new PET/CT machines. We currently use three different PET/CT machines. Phantom studies showed that SUVs obtained by an old PET/CT machine (Canon Aquiduo) were about 20-30\% (depending on the lesion size) lower than those obtained by new machines (Discovery 610 and IQ). The SUVs of Discovery 610 and Discovery IQ were equivalent. Therefore, we excluded the data obtained by Aquiduo PET/CT machine.

This study was only a single-institute retrospective study. The number of patients was not enough, and the interval between FDG studies was not fixed. There is no gold standard to evaluate skeletal metastases; therefore, we employed PERCIST or EORTC for bone as the comparative standard. As mentioned earlier, it is unclear whether this WS-TLG method can be applied to non-FDG avid skeletal metastasis.

This WS-TLG method consisted of automated organ (bone) segmentation from CT images and extraction of FDG-avid lesions using a cutoff level. Automated organ segmentation or extraction by CT images is now progressing rapidly [21]. Soon, FDG uptake of each organ (e.g., lung, liver, or etc.) can be separately evaluated. We hope that our study of WS-TLG is the beginning of organ extraction and tracer uptake evaluation in a clinical setting.

\section{Conclusion}

We have developed a semi-automatic quantitative method (WS-TLG) to evaluate skeletal metastases using FDG-PET/ CT data. The cutoff value was decided using FDG-PET/ CT data of breast cancer patients with and without skeletal metastasis, and SUV $=4.0$ was the tentative cutoff value. Using the cutoff value, 97\% (34/35) sensitivity and 91\% (77/85) specificity were obtained. The WS-TLG method was applied to 15 breast cancer patients with skeletal metastases who underwent therapy. WS-TLG was compared with PERCIST or EORTC only for bone, and very good agreement $(15 / 15)$ was obtained. This quantitative WS-TLG method seems to be a good biomarker to evaluate skeletal metastasis in breast cancer patients. Further clinical studies are warranted to evaluate the clinical values of this new WS-TLG method.

\section{Conflict of interest}

All authors have no potential conflicts of interest to disclose.
Acknowledgements We thank staffs (technology staffs and physicians) of Department of Nuclear Medicine for taking and diagnosing FDG-PET/CT studies. We also thank physicians of Breast Center for ordering FDG-PET/CT studies. We thank Ms. Hiromi Ito for editing this manuscript.

Funding None.

Open Access This article is distributed under the terms of the Creative Commons Attribution 4.0 International License (http://creativeco mmons.org/licenses/by/4.0/), which permits unrestricted use, distribution, and reproduction in any medium, provided you give appropriate credit to the original author(s) and the source, provide a link to the Creative Commons license, and indicate if changes were made.

\section{References}

1. Koizumi M, Yoshimoto M, Kasumi F, Iwase T. An open cohort study of bone metastasis incidence following surgery in breast cancer patients. BMC cancer. 2010;10:381.

2. Hamaoka T, Madewell JE, Podoloff DA, Hortobagyi GN, Ueno $\mathrm{N}$. Bone imaging in metastatic breast cancer. J Clin Oncol. 2004;22:2942-53.

3. Yang HL, Lui T, Wang XM, Xu Y, Deng SM. Diagnosis of bone metastases: a meta-analysis comparing 18FDG PET, CT, MRI and bone scintigraphy. Eur Radiol. 2011;21:2604-17.

4. Koizumi M, Matsumoto S, Takahashi S. T Yamashita, Ogata E: Bone metabolic markers are useful in the diagnosis of bone scan flare phenomenon in bone metastases from breast cancers. Clin Nucl Med. 1999;24:15-20.

5. Nakajima K, Nakajima Y, Horikoshi H, Ueno M, Wakabayashi $\mathrm{H}$, Shiga T, et al. Enhanced diagnostic accuracy for quantitative bone scan using an artificial neural network system: a Japanese multi-center database project. EJNMMI Res. 2013;3:83.

6. Koizumi M, Motegi K, Koyama M, Terauchi T, Yuasa T, Yonese J. Diagnostic performance of a computer-assisted diagnosis system for bone scintigraphy of newly developed skeletal metastasis in prostate cancer patients: search for low-sensitivity subgroups. Ann Nucl Med. 2017;31:521-8.

7. Mitsui Y, Shiina H, Yamamoto Y, Haramoto M, Arichi N, Yasumoto $\mathrm{H}$, et al. Prediction of survival benefit using an automated bone scan index in patients with castration-resistant prostate cancer. BJU int. 2012;110:628-34.

8. Reza M, Bjartell A, Ohlsson M, Kaboteh R, Wollmer P, Edenbrandt $\mathrm{L}$, et al. Bone Scan Index as a prognostic imaging biomarker during androgen deprivation therapy. EJNMMI Res. 2014;4:58.

9. Etchebehere EC, Araujo JC, Fox PS, Swanston NM, Macapinlac HA, Rohren EM. Prognostic factors in patients treated with 223Ra: the role of skeletal tumor burden on baseline $18 \mathrm{~F}-\mathrm{flu}$ oride PET/CT in predicting overall survival. J Nucl Med. 2015;56:1177-84.

10. Umeda T, Koizumi M, Fukai S, Miyaji N, Motegi K, Nakazawa $\mathrm{S}$, et al. Evaluation of bone metastatic burden by bone SPECT/CT in metastatic prostate cancer patients: defining threshold value for total bone uptake and assessment in radium-223 treated patients. Ann Nuc Med. 2018;32:105-13.

11. Tateishi U, Gamez C, Dawood S, Yeung HWD, Cristofanilli M, Macapinlac HA. Bone metastases in patients with metastatic breast cancer: Morphologic and metabolic monitoring of response to systemic therapy with integrated PET/CT. Radiology. 2008;247:189-96. 
12. Sugihara T, Koizumi M, Koyama M, Terauchi T, Gomi N, Ito Y, et al. Bone metastases from breast cancer: associations between morphologic CT patterns and glycolytic activity on PET and bone scintigraphy as well as explorative search for influential factors. Ann Nucl Med. 2017;31:719-25.

13. Chowdhury B, Sjostrom L, Alpsten M, Kostanty J, Kvist H, Lofgren R. A multicompartment body composition technique based on computerized tomography. Int J Obes Metab Disord. 1994;18:219-34.

14. Koizumi M, Yoshimoto M, Kasumi F, Ogata E. Differences between solitary and multiple bone metastases of breast cancer. Ann Oncol. 2003;14:1234-40.

15. Wahl RL, Jacene H, Kasamon Y, Lodge MA. From RECIST to PERCIST: evolving considerations for PET response criteria in solid tumors. J Nucl Med. 2009;50:122S-50S.

16. Young H, Baum R, Cremerius U, Herholz K, Hoekstra O, Lammertsma AA, et al. Measurement of clinical and subclinical tumour response using $[18 \mathrm{~F}]$-fluorodeoxyglucose and positron emission tomography: review and 1999 EORTC recommendations. European organization for research and treatment of cancer (EORTC) PET study group. Eur J Cancer 1999; 35:1773-1782.

17. Skougaard K, Nielsen D, Jensen BV, Hendel HW. Comparison of EORTC criteria and PERCIST for PET/CT response evaluation of patients with metastatic colorectal cancer treated with irinotecan and cetuximab. J Nucl Med. 2013;54:1-7.

18. Eisenhauer EA, Therasse P, Bogaerts J, Schwartz LH, Sargent D, Ford R, et al. New response evaluation criteria in solid tumours: revised RECIST guideline (version 1.1). Eur J Cancer 2009; 45:228-247.

19. Costelloe CM, Chuang HH, Madewell JE, Ueno NT. Cancer response criteria and bone metastases: RECIST 1.1, MDA and PERCIST. J Cancer 2010; 1:80-92.

20. Nakai T, Okuyama C, Kubota T, Yamada K, Ushijima Y, Taniike $\mathrm{K}$, et al. Pitfalls of FDG-PET for the diagnosis of osteoblastic bone metastases in patients with breast cancer. Eur J Nucl Med Mol Imaging. 2005;32:1253-8.

21. Gibson E, Giganti F, Hu Y, Bonmati E, Bandula S, Gurusamy K, et al. Automatic multi-organ segmentation on abdominal CT with dense V-networks. IEEE Trans Med Imaging. 2018;37:1822-34.

Publisher's Note Springer Nature remains neutral with regard to jurisdictional claims in published maps and institutional affiliations. 